\title{
Particle acceleration linked to Alfvén wave propagation on small scale density gradients
}

\author{
V. Génot ${ }^{1, *}$, F. Mottez ${ }^{2}$, and P. Louarn ${ }^{1}$ \\ ${ }^{1}$ CESR/CNRS, Toulouse, France \\ *Present address Queen Mary \& Westfield College, London, United Kingdom \\ ${ }^{2}$ CETP/CNRS, Vélizy, France
}

Camera-ready Copy for

Physics and Chemistry of the Earth

Manuscript-No. p1-11 


\title{
Particle acceleration linked to Alfvén wave propagation on small scale density gradients
}

\author{
V. Génot ${ }^{1, *}$, F. Mottez ${ }^{2}$, and P. Louarn ${ }^{1}$ \\ ${ }^{1}$ CESR/CNRS, Toulouse, France \\ *Present address Queen Mary \& Westfield College, London, United Kingdom \\ ${ }^{2}$ CETP/CNRS, Vélizy, France
}

Received 30 November 1999 - Accepted 30 June 2000

\begin{abstract}
We study how Alfvén waves propagate in the presence of sharp density gradients in the direction perpendicular to the ambient magnetic field. A fully electromagnetic electron guiding centre code is used for the simulation. During the propagation, initially parallel $\left(k_{\|}=0\right)$, transverse scales of the order of $c / \omega_{p e}$ are quickly reached which contributes to the creation of a significant parallel component of the electric field in the region of density inhomogeneity. The effects of this field on the velocity distribution functions are then discussed. In particular, we show that they can present a strong deviation from their initial Gaussian shape (global shift in energy) due to the action of the parallel electric field. Evidences are then given for a net energy gain of the electrons, to the expense of the wave, during this process. This energy transfer mechanism may be relevant in order to explain the particle acceleration in the auroral plasma cavities.
\end{abstract}

\section{Introduction}

Although the mean density of the auroral plasma is very small (a few $\mathrm{cm}^{-3}$ ), it presents a high degree of inhomogeneity. Following the results of Viking (e.g. Hilgers et al., 1992), the recent measurements of the Fast satellite have shown that spatial scales of the density variation shorter than the electron inertial length $\left(c / \omega_{p e}\right)$ can be encountered (Strangeway et al., 1998). Such structures have been named auroral plasma cavities. A consistent literature has reported that they are involved in many auroral processes from Auroral Kilometric Radiation generation to electrostatic turbulence (e.g. Louarn et al., 1990; Hilgers, 1992; Carlson et al., 1998). Recently Génot et al. (1999) have shown that they could play a major role in the acceleration of particles (up to energy of about a few keV). We studied how the characteristics of an Alfvén wave are modified during its propagation in a density cavity; it was shown that a non vanishing component of the electric field parallel to the ambient magnetic field progressively

Correspondence to: V. Génot (v.genot@qmw.ac.uk) forms, similarly to the Inertial Alfvén wave/Kinetic Alfvén wave model. This is briefly described in section 2 of the present article. The action of these waves on the particles is the main theme of the present study. Previously, using a model of parallel electric field (with a fixed $k_{\perp}$ ), Kletzing (1994) has studied the modification of electron distribution functions, whereas recently, Clark and Seyler (1999) investigated the formation of electron beam consecutive to the non linear evolution of an Inertial Alfvén Wave (IAW), thanks to a 1D self-consistent simulation. These two imposed conditions (a priori parallel electric field model and initial obliqueness of the wave) are released in our model to be computed self-consistently. Here, we present 2D Particle In Cell (PIC) simulations of a situation equivalent to the one of Génot et al. (1999), and particularly focus on the velocity distribution functions of the electrons that are modified by the presence of the parallel electric field formed on the density gradients. This behaviour is studied in section 3. Finally, we present some preliminary results about the effective dissipation of the incident wave.

\section{Parallel electric field formation}

From the theoretical point of view, since pioneering works (e.g. Hasegawa and Chen, 1975; Goertz, 1984), it is well known that the role of small spatial scales is crucial for the formation of parallel electric fields. Depending on the plasma $\beta$, retaining the transverse variation of the fields $\left(k_{\perp}\right)$ in comparison with either the electron inertial length $\left(c / \omega_{p e}\right.$, for $\beta<1$ ), or the ion Larmor radius $\left(\rho_{i}\right.$, for $\beta>1$ ), leads to a modification of the plasma dispersion relation. Note that in the second case, the characteristic length is in fact $c v_{t h e} / V_{A} \omega_{p e}$ which equals $\rho_{i}$ for $T_{i}=T_{e}$; in both cases the process effectively relies on the electron dynamics. It appears then that the electric field of the Alfvén wave can exhibit a parallel component. For the collisionless and very magnetized auroral plasma, this corresponds to the case of the IAW. Auroral plasma values lead for an Alfvén wave to 
a typical ratio $E_{\|} / E_{\perp} \simeq 0.001$ for the two components of the wave electric field. In their work, Génot et al. (1999) have shown that retaining the density $(n)$ variations in the direction perpendicular to the magnetic field $(x)$ leads to an additional term in the equations for the parallel electric field. As soon as this term is preponderant (that is $\frac{1}{n} \frac{\partial n}{\partial x} \geq k_{\perp}$ ) the value of the ratio $E_{\|} / E_{\perp}$ can be significantly larger than the one for the IAW. This was applied to the case of the auroral plasma cavities for which the typical density varies from a factor two to five on a few kilometers. An analytically simplified linear model has been established which describes the electric field behaviour when the Alfvén wave propagates into the cavity. The density variations with altitude come from a fit to in situ measurements, and the background magnetic field is dipolar. The convergence of magnetic field lines is effectively taken into account, as it is an important feature for auroral regions: the typical transverse scale indeed narrows from a factor $\sim 4$ between 20000 and $2000 \mathrm{~km}$. In the transverse direction, the density variations are modelled by an analytical expression which reproduce the variations inferred from Viking data. Thus, although the model is physically simplified (low frequency and cold plasma approximations), the typical quantities variations are realistic which enables to draw some relevant conclusions on the importance of plasma cavities in the acceleration mechanism. This model has been solved numerically for an initial parallel propagation $\left(k_{\perp}=0\right)$. The main results is that when the density gradients are steep enough such that they scale on $\sim c / \omega_{p e}$, the parallel electric field can reach values up to $\sim 10$ times the ones derived from the IAW model. These values maximizes on the locus of the density gradients where the wave front of the perpendicular component are also the more distorted $\left(k_{\perp} \neq 0\right)$. This is shown on Fig. 1 which presents a zoom on the simulation domain when the situation has evolved from the initial condition for which $E_{\|}=0$. The density contours have been superimposed on the parallel electric field plotted in colour code. The highest values are reached where these contours are the closest in the perpendicular direction (maximum gradient).

The physical mechanism is explain below. In the direction perpendicular to the magnetic field, the ions move following the polarization drift $\left(j_{x}=\frac{m_{i} n_{i}}{B^{2}} \frac{\partial E_{x}}{\partial t}\right.$, which is negligible for the electrons). On the density gradient locus, this induces the formation of a space charge. To counter this deviation to electric neutrality, in the low $\beta$ plasma that characterizes the auroral plasma (and our simulations), a parallel component of the electric field forms, on large parallel distances; the electrons then react promptly by being accelerated.

Following this linear study, we considered a more complete simulation able to tackle the non-linear plasma response. The aim is mainly to study the strong dissipation which is believed to take place in an inhomogeneous medium, such as the auroral cavity. This has been achieved thanks to a new implicit 2D PIC code which treats the ions kinetically whereas the electron motion are averaged over the gyro-period (for further details on this code the reader should refer to Mottez et al. (1998)). The results, presented extensively in

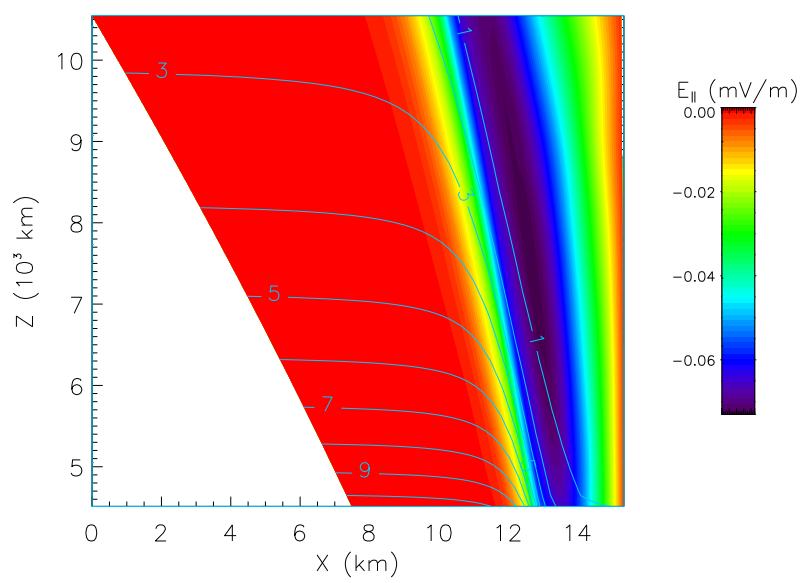

Fig. 1. Density and parallel electric field in a gradient region, computed from the analytical linear model of Génot et al. (1999), and as functions of both the altitude $(z)$ and the transverse coordinate $(x)$. The convergence of the magnetic field line is taken into account. The parallel electric field is shown when the Alfvén wave has propagated down to $\sim 4000 \mathrm{~km}$ in the cavity. The initial conditions would present the same density and no parallel electric field.

Génot et al. (In press), confirm the first linear study about the formation of parallel electric field on the density gradients (see Fig. 3 and explanations in the following section). During these simulations, ratios $E_{\|} / E_{\perp}$ of $\sim 0.01$ have been reached. With such high values of the parallel component of the electric field, one can readily address the question about how the plasma distribution is affected, especially the light electrons which are very sensitive to the parallel electric field. The kinetic nature of the code enables to display the distribution functions which is done in the following section.

\section{Parallel electric field effects on the particle distribu- tions}

The general conditions for the simulations described in this section, are the following: about $4.10^{6}$ particles are contained in a simulation box of $2048 \times 32$ cells, which physically represents about $1000 \mathrm{~km}$ in the direction parallel to the magnetic field $(z)$ and $15 \mathrm{~km}$ in the perpendicular direction $(x)$. The boundary conditions are periodic in both directions. In the middle of the box the density is decreased by a factor of 4 . It thus presents a channel-like configuration. We study the temporal evolution of a linearly polarized wave (superposition of an Alfvén wave and a fast magnetosonic wave, whose wavelengths equal the size of the box) over a few Alfvén periods. The Alfvén speed is $0.4 c$.

To have a first insight of the direct effect of the plasma inhomogeneities on the particles, we conducted a comparison test. The same wave was launched in two simulations with different density configuration: the first presented a channel as described above, whereas the second was uniform. We selected a set of particles, initially from the gradient region (for the inhomogeneous case) and followed their evolution in the 

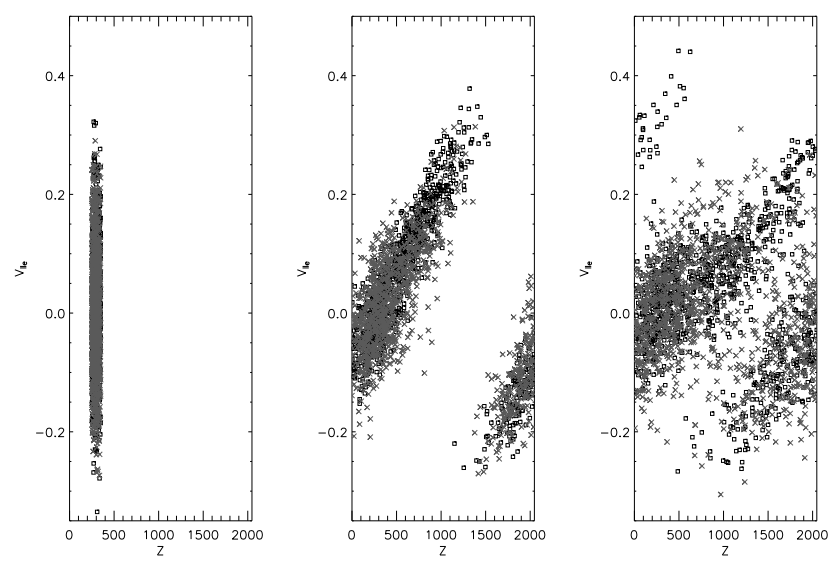

Fig. 2. Evolution of the electron velocity distribution function seen in PIC simulations. Symbols correspond to macro-electrons: crosses for the simulation with an uniform density, squares for the simulation with an inhomogeneous density. The distance and the velocity are normalized to $0.1 c / \omega_{p e}$ and $c$ respectively.

phase space $\left(z, v_{\|}\right)$. The results are shown on Fig. 2.

As time is running, the particles move in the $z$ direction depending on their initial parallel velocity. However, some of them encounter a region of parallel electric field and get accelerated. This only appears in the inhomogeneous case, as it can be seen on the third panel: the particles located in the zone $\left(0<Z<500,0.25<V_{\| e}<0.45\right)$ for this time only exist in the presence of the density gradient. These particles may thus be resonant particles interacting with the parallel component of the electric field which travels at the Alfvén velocity. Although the particles come initially from the density gradient region, they do not all encounter a parallel electric field. Indeed, the particles get dispersed from their original position to the entire simulation box due to their parallel velocity, but the parallel electric field forms locally, where the perpendicular component and thus the polarization drift is the largest. The consequence is that the distribution function, for this set of particles, integrated over all $z$ presents a beam located at $v_{\|} \simeq 0.35 c$ superimposed to the Gaussian distribution of non accelerated particles.

On the contrary, distribution functions of particles strictly localized on density gradient locus where $E_{\|} \neq 0$ exhibit rather different characteristics. To compute such distributions we isolate, at a given time, a region with a parallel electric field extremum, typically a rectangle in the simulation box, and we retain all the particle phase space coordinates. This is done on Fig. 3: the computation region is superimposed on the colour level plot of the parallel electric field. The resulting distribution function is plotted on Fig. 4.

One notes a complete shift of the function (that now peaks at $0.05 c$ ) which denotes a global response of the electron bulk to the accelerating parallel electric field. Such a distortion of the distribution functions suggests that the light electrons immediately react to the parallel electric field applied. This quick time response may also explain why nothing significant appears on the ions, neither perpendicular heating nor

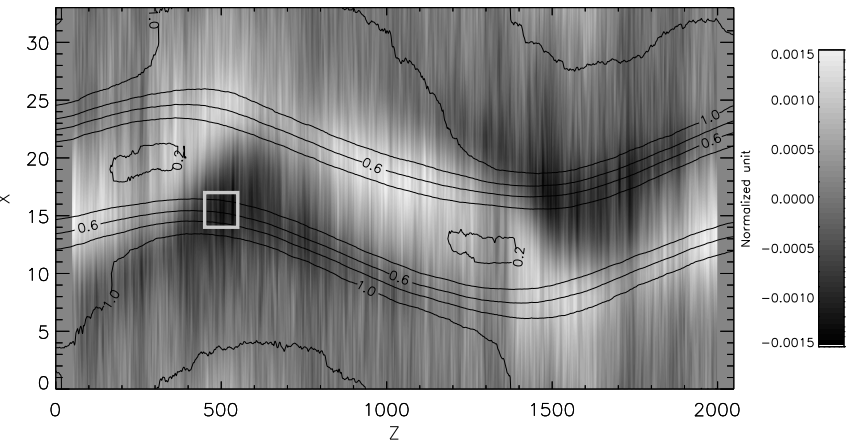

Fig. 3. Parallel electric field computed self-consistently with a PIC code (in unit of $\omega_{p e} c m_{e} / e$ ). The electron density contours have been superimposed. The extremum arise on the density gradient locus. The white box bounds the region where the velocity distribution function shown on Fig. 4 is computed.

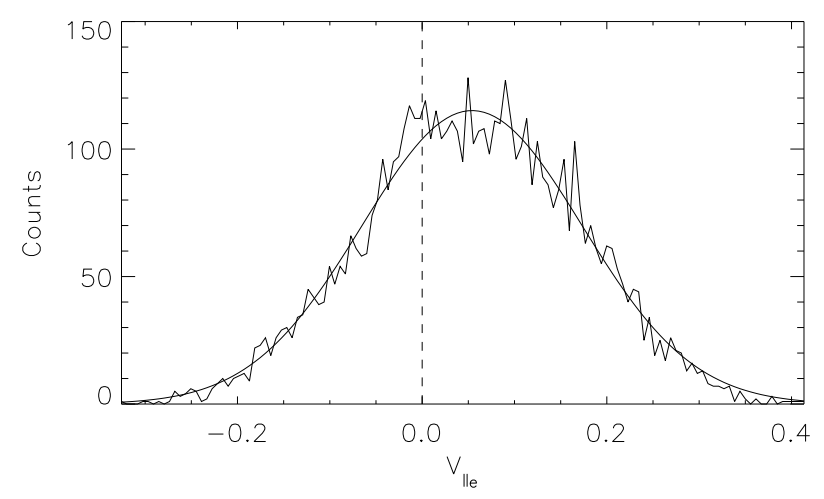

Fig. 4. Electron velocity distribution function computed in a region with a parallel electric field extremum (see the white box on Fig. 3). The deviation from the zero-centred shape denotes the bulk acceleration of the electrons. 

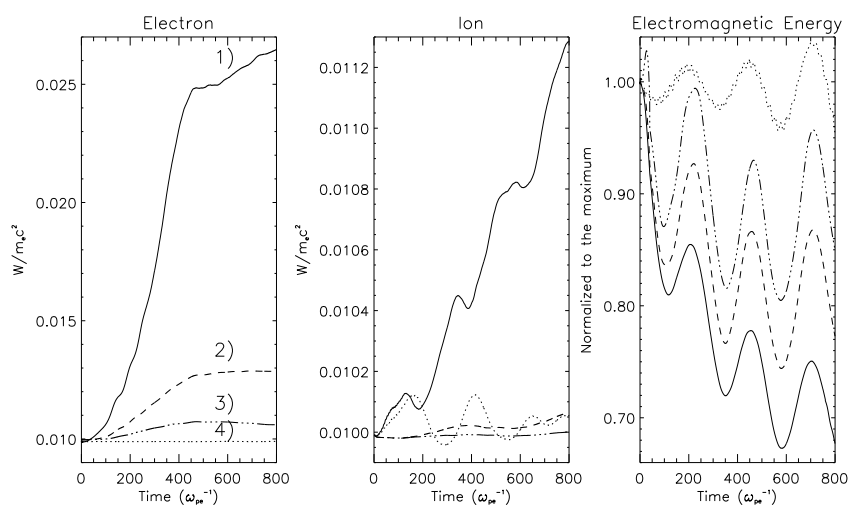

Fig. 5. Electron and ion parallel kinetic energies and electromagnetic energy versus time. The curves correspond to simulations with different levels of the initial wave amplitude : 1) $\left.\delta B / B_{0}=0.05,2\right) \delta B / B_{0}=0.02,3$ ) $\left.\delta B / B_{0}=0.01,4\right)$ uniform density and $\delta B / B_{0}=0.05$.

direct acceleration by the parallel electric field. This is confirmed by the energy diagnostics of the following section.

\section{Energy transfer}

In this section, we draw preliminary results about the significant energy transfer from the waves to the particles. This is an attempt to explain the strong dissipation of the Alfvén wave which is believed to take place in the auroral region. Fig. 5 presents the temporal variations of the parallel kinetic energy of both electrons and ions, as well as the temporal variation of the electromagnetic energy. The different curves correspond to different amplitudes of the initial perturbation with or without perpendicular density variation. We see that the more energy is injected into the system, the more it decreases with time whereas the parallel kinetic energies of the particles are enhanced.

This denotes a net transfer of energy from the wave to the particles. However, the non linearity of the process is a not yet well understood feature. The electrons see their parallel kinetic energy multiplied by a factor 2.5 (there is only a factor 1.12 for the ions). The point we want to emphasize here is that such a dissipation of the wave is directly linked to the existence of the density gradients. As pointed out in Fig. 5, case 4, there is neither dissipation nor acceleration in the case of a homogeneous density. The density gradients may thus be ideal locations for both processes of dissipation and particle acceleration by propagating Alfvén waves. This could be a suggested mechanism to explain the brightness of the auroral arc: the whole structure of the arc is related to the acceleration region (the density cavity) but the brightest part may be connected to the density gradient locus where particles are preferentially accelerated.

\section{Conclusions}

Improving the results of the linear model, these kinetic simulations show several key features of the physics of MHD waves in inhomogeneous plasmas. It is in particular relevant to the case of the auroral plasma cavities that exhibit strong density gradients in the direction perpendicular to the background magnetic field. The wave spectrum of an incoming Alfvén wave (initially in pure parallel propagation) cascades to small length scales $\left(\sim c / \omega_{p e}\right)$ by a linear process (the progressive distortion of the wavefront). Simultaneously large parallel electric fields arise on the density gradient locus. The electrons are particularly sensitive to this field, and in the region of its extremum, react globally and very dynamically, which results in a complete shift of their velocity distribution functions. The energy transfer is clearly in favour of the electrons that experience a doubling of their parallel kinetic energy over one Alfvén period. All these features are proved to be linked to the existence of plasma inhomogeneities, which emphasizes the importance of plasma cavity as regards of many auroral physics phenomena.

Acknowledgements. The numerical computations have been supported by the CNRS and were made on the C98 at the IDRIS computation center (France).

\section{References}

Carlson, C.W. et al., Fast observations in the downward auroral current region: Energetic upgoing electron beams, parallel potential drops, and ion heating, Geophys. Rev. Lett. 25, , 2017, 1998.

Clark, A. E., and C. E. Seyler, Electron beam formation by small-scale oblique inertial Alfvén waves, J. Geophys. Res. 104, 17233, 1999.

Génot, V., P. Louarn, and D. Le Quéau, A study of the propagation of Alfvén waves in the auroral density cavities, J. Geophys. Res. 104, 22649, 1999.

Génot, V., F. Mottez, and P. Louarn, Electron acceleration by Alfvén waves in density cavities, In press, J. Geophys. Res..

Goertz, C.K., Kinetic Alfvén waves on auroral field lines, Planet. Space Sci., 32, 11, 1387, 1984.

Hasegawa, A., and L. Chen, Kinetic process of plasma heating due to Alfvén wave excitation, Phys. Rev. Lett., 35, 370, 1975.

Hilgers, A., The auroral radiating plasma cavities, Geophys. Rev. Lett. 19, 237, 1992.

Hilgers, A., B. Holback, G. Holmgren, and R. Boström, Probe measurements of low plasma densities with application to the auroral acceleration regions and auroral kilometric radiation sources, J. Geophys. Res. 97, 8631, 1992.

Kletzing, C. A., Electron acceleration by kinetic Alfvén waves, J. Geophys. Res. 99, 11095, 1994.

Louarn, P., A. Roux, H. de Féraudy, D. Le Quéau, M. André, and L. Matson, Trapped electrons as a free energy source for the auroral kilometric radiation, J. Geophys. Res. 95, 5983, 1990.

Mottez, F., J.C. Adam, and A. Heron, A new guiding centre PIC scheme for electromagnetic highly magnetized plasma simulation, Computer Physics Communication, 113, 1-22, 1998.

Strangeway, R. J. et al., Fast observations of VLF waves in the auroral zone: Evidence of very low plasma densities, Geophys. Rev. Lett. 25, 2065, 1998. 